\title{
SHARP LOWER AND UPPER BOUNDS FOR THE $q$-GAMMA FUNCTION
}

\author{
AHMED SALEM
}

Abstract. This paper is devoted to provide sharp bounds for the $q$-gamma function from below and above for all $q>0$ by means of investigating the monotonicity property to analytical functions involving logarithm $q$-gamma function. It turns out that these results refine and improve lower and upper bounds for the $q$-gamma function which have been given by Salem [13].

Mathematics subject classification (2010): 33D05, 26D07, 26A48.

Keywords and phrases: Inequalities, $q$-Gamma function, $q$-digamma functions, monotonic function.

\section{REFERENCES}

[1] M. Abramowitz and I. A. Stegun (EDS), Handbook of Mathematical Functions with Formulas, Graphs, and Mathematical Tables, National Bureau of Standards, Applied Mathematics Series 55, 9th printing, Washington, 1970.

[2] F. AlZahrani And A. SAlem, Sharp bounds for the Lambert $W$ function, Integr. transf. Spec. F., 2912 (2018), 971-978.

[3] H. AlZer AND A.Z. GRinshPAn, Inequalities for the gamma and q-gamma functions, J. Approx. Theory, 144 (2007), 67-82.

[4] N. BATIR, Monotonicity properties of q-digamma and q-trigamma functions, J. Approx. Theory, 192 7 (2015), 336-346.

[5] N. BATIR, q-Extensions of some estimates associated with the digamma function, J. Approx. Theory, 174 (2013), 54-64.

[6] N. Elezovic, C. Giordano and J. Pecaric, Convexity and q-gamma function, Rendiconti del Circolo Matematico di Palermo, Serie II, Tomo XLVIII (1999) 285-298.

[7] P. GAO, Some Monotonicity Properties of Gamma and q-Gamma Functions, ISRN Math. Anal., 2011 (2011), 1-15.

[8] A.Z. GRINShPAN AND M.E.H. IsmaiL, Completely monotonic functions involving the gamma and q-gamma functions, Proc. Amer. Math. Soc., 134 (2006), 1153-1160.

[9] M. E. H. Ismail AND M. E. Muldoon, Inequalities and monotonicity properties for gamma and q-gamma functions, in: R.V.M. Zahar (Ed.), Approx. Comput., Inter. Series of Numerical Math., 119 Birkhauser, Boston, MA (1994), 309-323.

[10] C. KRATtenthaler AND H. M. SRIVASTAVA, Summations for basic hypergeometric series involving a q-analogue of the digamma function, Computers Math. Appl., 322 (1996), 73-91.

[11] D.S. MOAK, The q-gamma function for $q>1$, Aequationes Math., 20 (1980), 278-285.

[12] D.S. MoAK, The q-analogue of Stirling's formula, Rocky Mountain J. Math., 14 (1984) 403-413.

[13] A. SALEM, Monotonic functions related to the q-gamma function, Monatsh. Math., 179, 2 (2016), 281-292.

[14] A. SALEM, Sharp bounds for the q-gamma function in terms of the Lambert $W$ function, Ramanujan J., 492 (2019), 321-339.

[15] A. Salem And F. Alzahrani, Complete monotonicity property for two functions related to the q-digamma function, J. Math. Inequal., 131 (2019), 37-52.

[16] A. SALEM, Some inequalities for the q-gamma and the q-polygamma functions, Thai J. Math., 163 (2018), 683-692.

[17] A. SAlem AND F. AlZAhrani, Improvements of bounds for the q-gamma and the q-polygamma functions, J. Math. Inequal., 113 (2017), 873-883. 
[18] A. SALEM, Completely monotonic functions related to the gamma and the q-gamma functions, Rev. R. Acad. Cienc. Exactas Fs. Nat. Ser. A Mat. RACSAM, 111 (2017), 271-280.

[19] A. SALEM, Some classes of completely monotonic functions related to q-gamma and q-digamma functions, Math. Inequal. Appl., 193 (2016), 853-862.

[20] A. SALEM, A certain class of approximations for the q-digamma function, Rocky Mountain J. Math., 465 (2016), 1665-1677.

[21] A. SAlEM, Completely monotonic functions related to the q-gamma and the q-trigamma functions, Anal. Appl., 132 (2015), 125-134.

[22] A. SALEM, Complete monotonicity properties of functions involving q-gamma and q-digamma functions, Math. Inequal. Appl., 173 (2014), 801-811.

[23] A. SALEM, An infinite class of completely monotonic functions involving the q-gamma function, J. Math. Anal. Appl., 406 (2013), 392-399.

[24] A. SALEM, A completely monotonic function involving q-gamma and q-digamma functions, J. Approx. Theory, 1647 (2012), 971-980.

[25] A. Salem, Some Properties and Expansions Associated with q-Digamma Function, Quaest. math., 361 (2013), 67-77. 